\title{
Long-term follow-up of neurosurgical outcomes for adult patients in Uganda with traumatic brain injury
}

\author{
*Michael C. Jin, BS, ${ }^{1}$ Bina Kakusa, BS, ${ }^{1}$ Seul Ku, MS, ${ }^{1}$ Silvia D. Vaca, MD, ${ }^{2}$ Linda W. Xu, MD, ${ }^{3}$ \\ Juliet Nalwanga, MBChB, MMed Surg, ${ }^{4}$ Joel Kiryabwire, MBChB, MMed Surg, ${ }^{4}$ \\ Hussein Ssenyonjo, MBChB, MMed Surg, ${ }^{4}$ John Mukasa, MBChB, MMed Surg, ${ }^{4}$ \\ Michael Muhumuza, MBChB, MMed Surg, ${ }^{4}$ Anthony T. Fuller, MD, MSc-GH, ${ }^{5,6}$ \\ Michael M. Haglund, MD, PhD, MACM, ${ }^{5,6}$ and Gerald A. Grant, MD² \\ ${ }^{1}$ School of Medicine and 2Department of Neurosurgery, Stanford University, Palo Alto, California; ${ }^{3}$ Department of Neurosurgery, \\ Allegheny Medical Group, Pittsburgh, Pennsylvania; ' ${ }^{2}$ Department of Neurosurgery, Mulago National Referral Hospital, Kampala, \\ Uganda; ${ }^{5}$ Department of Neurosurgery, Duke University, Durham, North Carolina; and ${ }^{6}$ Duke Global Neurosurgery and \\ Neurology, Durham, North Carolina
}

OBJECTIVE Traumatic brain injury (TBI) is a major cause of mortality and morbidity in Uganda and other low- and middle-income countries (LMICs). Due to the difficulty of long-term in-person follow-up, there is a paucity of literature on longitudinal outcomes of TBI in LMICs. Using a scalable phone-centered survey, this study attempted to investigate factors associated with both mortality and quality of life in Ugandan patients with TBI.

METHODS A prospective registry of adult patients with TBI admitted to the neurosurgical ward at Mulago $\mathrm{National} R e$ ferral Hospital was assembled. Long-term follow-up was conducted between 10.4 and 30.5 months after discharge (median 18.6 months). Statistical analyses included univariable and multivariable logistic regression and Cox proportional hazards regression to elucidate factors associated with mortality and long-term recovery.

RESULTS A total of 1274 adult patients with TBI were included, of whom 302 (23.7\%) died as inpatients. Patients who died as inpatients received surgery less frequently $(p<0.001)$, had more severe TBI at presentation $(p<0.001)$, were older ( $p<0.001)$, and were more likely to be female $(p<0.0001)$. Patients presenting with TBI resulting from assault were at reduced risk of inpatient death compared with those presenting with TBI caused by road traffic accidents (OR $0.362,95 \% \mathrm{Cl} 0.128-0.933)$. Inpatient mortality and postdischarge mortality prior to follow-up were $23.7 \%$ and $9 \%$, respectively. Of those discharged, $60.8 \%$ were reached through phone interviews. Higher Glasgow Coma Scale score at discharge (continuous $\mathrm{HR} 0.71,95 \% \mathrm{Cl} 0.53-0.94$ ) was associated with improved long-term survival. Tracheostomy (HR 4.38, 95\% Cl 1.05-16.7) and older age (continuous HR 1.03, 95\% Cl 1.009-1.05) were associated with poor longterm outcomes. More than $15 \%$ of patients continued to suffer from TBI sequelae years after the initial injury, including seizures (6.1\%) and depression (10.0\%). Despite more than $60 \%$ of patients seeking follow-up healthcare visits, mortality was still $9 \%$ among discharged patients, suggesting a need for improved longitudinal care to monitor recovery progress.

CONCLUSIONS Inpatient and postdischarge mortality remain high following admission to Uganda's main tertiary hospital with the diagnosis of TBI. Furthermore, posttraumatic sequelae, including seizures and depression, continue to burden patients years after discharge. Effective scalable solutions, including phone interviews, are needed to elucidate and address factors limiting in-hospital capacity and access to follow-up healthcare.

https://thejns.org/doi/abs/10.3171/2020.4.JNS193092

KEYWORDS follow-up; LMIC; low- and middle-income countries; trauma; TBI; Uganda; traumatic brain injury

ABBREVIATIONS GCS = Glasgow Coma Scale; GOSE = Extended Glasgow Outcome Scale; HICs = high-income countries; IPQ-9 = Brief IIIness Perception Questionnaire; LMICs = low- and middle-income countries; LOS = length of stay; MNRH = Mulago National Referral Hospital; PHQ-9 = Patient Health Questionnaire-9; PTE = posttraumatic epilepsy; RTA = road traffic accident; SF-12 = 12-Item Short-Form Health Survey; TBI = traumatic brain injury.

SUBMITTED November 13, 2019. ACCEPTED April 6, 2020.

INCLUDE WHEN CITING Published online July 3, 2020; DOI: 10.3171/2020.4.JNS193092.

${ }^{*}$ M.C.J. and B.K. contributed equally to this work. 
$\mathrm{T}$ RAUMATIC brain injury (TBI) remains one of the leading causes of mortality and morbidity in lowand middle-income countries (LMICs). ${ }^{1-4}$ In addition to preadmission and inpatient mortality, disability and neurological trauma attributable to TBI can have lasting effects on patients and their social networks. Patients with TBI in high-income countries (HICs) likely benefit from well-established healthcare systems and public infrastructure providing longitudinal healthcare services, such as follow-up care and rehabilitation services, which are limited in LMICs. ${ }^{2,5}$ Among other consequences, this likely leads to higher rates and longer-lasting burden of post-TBI sequelae, which have been associated with depressed cognition and lowered quality of life. 6,7 The factors that influence long-term outcomes of patients with TBI residing in LMICs-where patient presentation, healthcare practices, and social infrastructure differ from $\mathrm{HICs}$ - remain understudied.

In Uganda, the incidence of TBI is 1.5 times greater than the global incidence (106/100,000), and head and neck injuries account for $65 \%$ of all injury-related deaths. ${ }^{3,8}$ Nearly $60 \%$ of TBI cases in Africa are attributable to road traffic accidents (RTAs), ${ }^{9}$ compared with only $25 \%$ in North America. Access to neurosurgical care in Uganda remains a barrier, resulting in a higher proportion of patients undergoing nonoperative treatment. ${ }^{10,11}$ In one study, of the $23 \%$ of patients with TBI admitted for surgery at Mulago National Referral Hospital (MNRH), Uganda's main tertiary care center, nearly $22 \%$ failed to receive surgical care. ${ }^{12}$

In the past decade, there have been efforts to improve neurosurgical care in Uganda. ${ }^{13,14}$ As healthcare capacity expands, it becomes increasingly important to elucidate optimal neurosurgical practices. Furthermore, Uganda's continued population and road congestion growth ${ }^{15,16}$ portend potential increases in RTAs, further necessitating studies expanding upon TBI management. ${ }^{9}$ We sought to identify factors associated with inpatient mortality and long-term patient recovery in a prospectively enrolled cohort at MNRH. Additionally, we sought to investigate the longitudinal impact of TBI on patients' quality of life. Because long-term follow-up in LMICs can be challenging due to a lack of established infrastructure, we utilized previously validated phone surveys to assess long-term recovery of neurocognitive function to understand the risk of subsequent TBI-related sequelae..$^{11,17,18}$

\section{Methods}

\section{Patient Population and Study Setting}

Adult patients with TBI referred to the neurosurgical ward at MNRH between 2014 and 2015 were prospectively recruited. Collected data variables at inpatient admission included age, sex, Glasgow Coma Scale (GCS) score at admission and discharge, intervention received (including mechanical ventilation, tracheostomy, and surgery), cause for admission, and inpatient hospital length of stay (LOS). For the subset of patients with available phone numbers, longitudinal follow-up was conducted between 10 and 30 months after discharge. Outcome variables included postdischarge mortality, neurological outcomes per the Extended Glasgow Outcome Scale (GOSE), ${ }^{19}$ and quality-of-life indicators.

Ethical approval for the study was provided by the Stanford University IRB in the US and the MNRH Research and Ethics Committee in Uganda.

\section{Data Collection}

Data collection was completed as previously described. ${ }^{11}$ Briefly, phone surveys were developed based on literature review, survey design, content expert review, and Ugandan collaborator review for adaptation to this setting. Surveys inquired about caregiver demographics, follow-up healthcare, morbidity and mortality, quality of life, and access to home health resources. Quality-of-life metrics, including functional independence, recurrent seizures, and frequency of depressive episodes, were also qualitatively assessed (for further elaboration, see Appendix). Calls were conducted in the participant's native language by Ugandan research staff. Follow-up inquiries were conducted by Ugandan research staff, who ensured patient consent prior to each call. Data were independently reviewed by research coordinators and staff to confirm data consistency and quality. If necessary, additional clarification was solicited in additional calls. In cases in which the patient died after discharge but prior to follow-up, surveys were conducted with available family members or caregivers.

\section{Statistical Analysis}

Descriptive statistics were calculated for demographics, mortality, and follow-up healthcare. Functional outcomes were assessed using the GOSE. ${ }^{20}$ Chi-square statistics were calculated to assess differences in categorical variables. Cells representing unknown values were not included in chi-square testing. Wilcoxon rank-sum and Kruskal-Wallis tests were used to compare distributions of continuous variables in a nonparametric manner. Logistic regression was used to evaluate factors influencing receipt of surgery and presence of neurological deficits at last follow-up. Cox proportional hazards regression was implemented to identify features associated with survival following patient discharge. For both logistic regression and Cox regression models, input covariates with a univariable $p$ value less than an a priori-defined threshold of 0.1 were included in multivariable analyses. TBI resulting from RTAs and assault were separately analyzed in additional analyses, as prior studies have indicated these etiologies are associated with unintentional and intentional TBI, respectively. ${ }^{16}$ Further subset analysis was conducted on the patients receiving surgery during initial TBI admission. All statistical tests were 2 -sided, and $\mathrm{p}$ values $<0.05$ were considered significant. Data analysis was completed using Microsoft Excel 2013 and the R statistical program (version 3.6.0, The R Project for Statistical Computing). Additional graphic analyses were performed using $\mathrm{R}$ and GraphPad Prism (version 8).

\section{Results}

\section{Inpatient Demographics}

Between 2014 and 2015, a total of 2032 patients were admitted to the neurosurgical ward at MNRH, of whom 
TABLE 1. Cohort characteristics

\begin{tabular}{|c|c|c|c|c|}
\hline Parameter & Inpatient Death & Discharged & p Value & Total \\
\hline No. of patients & 302 & 972 & & 1274 \\
\hline \multicolumn{5}{|l|}{ Patient demographics } \\
\hline Sex & & & $<0.0001$ & \\
\hline Female & $35(11.6)$ & $100(10.3)$ & & $135(10.6)$ \\
\hline Male & $261(86.4)$ & $872(89.7)$ & & $1133(88.9)$ \\
\hline Unspecified & $6(2.0)$ & $0(0.0)$ & & $6(0.5)$ \\
\hline Median age (range), yrs & $32(18-89)$ & $28(18-95)$ & $<0.0001$ & $29(18-95)$ \\
\hline $18-30$ & $83(27.5)$ & $567(58.3)$ & & $650(51)$ \\
\hline $31-45$ & $58(19.2)$ & $255(26.2)$ & & $313(24.6)$ \\
\hline $46-60$ & $25(8.3)$ & $86(8.8)$ & & $111(8.7)$ \\
\hline $61+$ & $20(6.6)$ & $47(4.8)$ & & $67(5.3)$ \\
\hline Unspecified & $116(38.4)$ & $17(1.7)$ & & $133(10.4)$ \\
\hline \multicolumn{5}{|l|}{ Injury characteristics } \\
\hline Type of injury & & & 0.060 & \\
\hline RTA & $141(46.7)$ & $540(55.6)$ & & $681(53.5)$ \\
\hline Assault & $63(20.9)$ & $301(31)$ & & $364(28.6)$ \\
\hline Fall & $4(1.3)$ & $47(4.8)$ & & $51(4)$ \\
\hline Other & $1(0.3)$ & $13(1.3)$ & & $14(1.1)$ \\
\hline Unspecified & $93(30.8)$ & $71(7.3)$ & & $164(12.9)$ \\
\hline Median admission GCS score (range) & $9(3-15)$ & $14(4-15)$ & $<0.0001$ & $13(3-15)$ \\
\hline \multicolumn{5}{|l|}{ Inpatient management } \\
\hline Median LOS (range), days & $2(0-142)$ & $5(0-110)$ & 0.72 & $4(0-142)$ \\
\hline Received surgery & $38(12.6)$ & $204(21)$ & 0.0007 & $242(19)$ \\
\hline Median discharge GCS score (range) & $3(3-3)$ & $15(6-15)$ & & $15(3-15)$ \\
\hline
\end{tabular}

$1274(62.7 \%)$ were adult patients presenting due to trauma (Table 1, Fig. 1).

Of those admitted to the neurosurgical ward, 242 (19\%) underwent surgery. Factors associated with receiving surgery were assessed in the subset of patients alive at discharge (Table 2). Older patients with TBI (continuous variable; OR 1.002 , 95\% CI 1.000-1.004), those with abnormal pupil examinations (OR 1.40, 95\% CI 1.19-1.63), and those with focal neurological deficits (OR 1.12, 95\% CI $1.03-1.23$ ) were more likely to receive surgery. Additionally, those who presented with non-RTA-associated TBI, compared with RTA-associated TBI (assault: OR 1.18, 95\% CI 1.12-1.25; fall/accident: OR $1.15,95 \%$ CI $1.02-$ 1.30; other causes: OR $1.59,95 \%$ CI 1.28-1.97), were more likely to receive surgery upon admission. Those with CSF leaks were less likely to receive surgery (OR $0.91,95 \%$ CI 0.85-0.98). Sex, geographic region, and admission GCS score were not associated with a proclivity to receive surgery. When assessing the likelihood of receiving surgery using GCS score stratified into mild, moderate, or severe TBI, presentation with severe TBI was independently associated with increased use of surgery (OR $3.03,95 \% \mathrm{CI}$ $1.23-7.31)$.

\section{Inpatient Outcomes}

Of the 1274 patients, 302 (23.7\%) died while inpatients

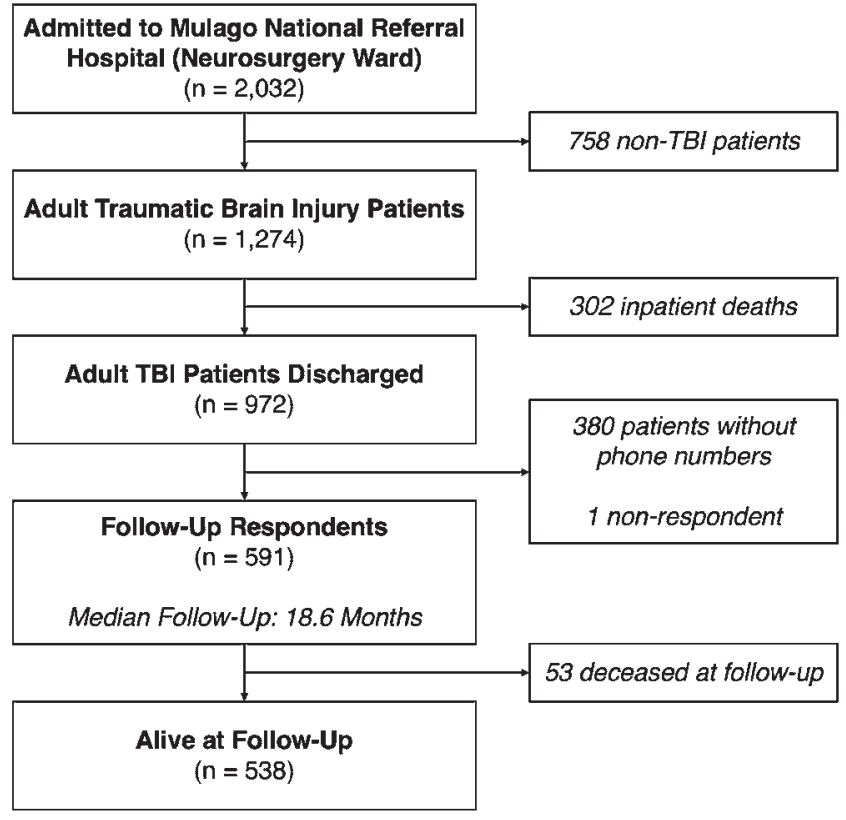

FIG. 1. CONSORT diagram. 
TABLE 2. Factors associated with receiving surgery

\begin{tabular}{|c|c|c|c|c|}
\hline \multirow[b]{2}{*}{ Parameter } & \multicolumn{2}{|c|}{ Univariable } & \multicolumn{2}{|c|}{ Multivariable } \\
\hline & OR $(95 \% \mathrm{Cl})$ & p Value & OR $(95 \% \mathrm{Cl})$ & $\mathrm{p}$ Value \\
\hline \multicolumn{5}{|l|}{ Sex } \\
\hline \multicolumn{5}{|l|}{ Female (ref) } \\
\hline Male & $1.04(0.95-1.14)$ & 0.384 & & \\
\hline Age (continuous variable) & $1.004(1.002-1.007)$ & $<0.001$ & $1.002(1.000-1.004)$ & 0.036 \\
\hline \multicolumn{5}{|l|}{ Region } \\
\hline \multicolumn{5}{|l|}{ Central (ref) } \\
\hline Eastern & $1.08(0.96-1.21)$ & 0.181 & & \\
\hline Northern & $1.09(0.92-1.28)$ & 0.325 & & \\
\hline Western & $0.97(0.88-1.06)$ & 0.495 & & \\
\hline \multicolumn{5}{|l|}{ Injury cause } \\
\hline \multicolumn{5}{|l|}{ RTA (ref) } \\
\hline Assault & $1.16(1.09-1.23)$ & $<0.001$ & $1.18(1.12-1.25)$ & $<0.001$ \\
\hline Fall & $1.21(1.07-1.36)$ & 0.003 & $1.15(1.02-1.30)$ & 0.021 \\
\hline Other & $1.73(1.38-2.15)$ & $<0.001$ & $1.59(1.28-1.97)$ & $<0.001$ \\
\hline Admission GCS score (continuous variable) & $1.00(0.99-1.01)$ & 0.345 & & \\
\hline Positive CSF leak (vs no leak) & $0.85(0.78-0.92)$ & $<0.001$ & $0.91(0.85-0.98)$ & 0.014 \\
\hline Abnormal pupil exam (vs normal exam) & $1.37(1.16-1.62)$ & $<0.001$ & $1.40(1.19-1.63)$ & $<0.001$ \\
\hline Focal neural deficits (vs none) & $1.21(1.10-1.34)$ & $<0.001$ & $1.12(1.03-1.23)$ & 0.013 \\
\hline
\end{tabular}

Boldface type indicates statistical significance.

(Table 1). There were no significant differences in LOS ( $p$ $=0.72$ ) in patients who died while inpatients versus those who survived to discharge. However, older patients $(\mathrm{p}<$ 0.001 ), patients with lower admission GCS scores ( $p<$ $0.001)$, and female patients $(\mathrm{p}<0.001)$ had higher rates of mortality. Patients who died while inpatients were less likely to have received surgical treatment $(\mathrm{p}<0.001)$.

In the 242 patients receiving surgery, the inpatient mortality rate was $15.7 \%(n=38)$. After adjusting for age, sex, injury type, and GCS score at admission, patients presenting with TBI due to assault were less likely to die as inpatients compared with those presenting with RTAassociated TBI (OR 0.362, 95\% CI 0.128-0.933). Patients presenting with moderate disability (admission GCS score 9-12) or severe disability (admission GCS score 3-8) were at a significantly increased risk for death following surgery compared with those presenting with mild disability (moderate disability: OR 4.640, 95\% CI 1.510-15.99; severe disability: OR 12.63, 95\% CI 4.348-42.72). Age and sex did not impact inpatient mortality in patients receiving surgery for TBI.

RTAs and assaults accounted for more than $80 \%$ of the neurosurgical ward admissions (total $\mathrm{n}=1045$ [82.0\%]: 681 RTAs, 364 assaults). RTA-associated TBI, compared with assault-associated TBI, was more frequently observed with older age (median age 30 vs 28 years, $p<0.001$ ) and male sex $(95.9 \%$ vs $86.9 \%, \mathrm{p}<0.001)$. Assault-associated TBI was more frequently treated with surgery than RTAassociated TBI $(25.8 \%$ vs $15.0 \%, \mathrm{p}<0.001)$. Tracheostomy and mechanical ventilation rates were similar regardless of TBI etiology. The length of inpatient stay was similar between RTA and assault patients (median 4 vs 5 days, $\mathrm{p}=0.468$ ); however, patients who underwent surgery for assault-associated TBI had longer inpatient stays than those with RTA-associated TBI (median 7 vs 5 days, $\mathrm{p}=$ 0.029). Improvements in patient condition, defined by an increase in GCS score from admission to discharge, were more frequent in patients admitted for RTA-associated than assault-associated TBI (RTA $59.4 \%$ vs assault $46.7 \%$, $\mathrm{p}<0.001)$. When restricted to patients receiving surgery, similar percentages of patients demonstrated neurological improvements (RTA $59.2 \%$ vs assault $54.1 \%, \mathrm{p}=0.623$ ). Neurological improvement was less frequent in nonsurgical assault patients than in nonsurgical RTA patients (assault $43.6 \%$ vs RTA $59.4 \%, \mathrm{p}<0.001)$.

\section{Follow-Up Demographics and Outcomes}

Of the 972 patients discharged, 591 (60.8\%) responded to follow-up calls (Table 3, Fig. 1). The median followup length was 18.6 months (range 10.4-30.5 months). Of the 591 respondents, $522(88.3 \%)$ were male, the median age was 28 years, $334(56.5 \%)$ had a primary education or less, and 486 patients were employed $(n=458,77.5 \%)$ or students $(\mathrm{n}=28,4.7 \%)$ at the time of follow-up. The majority of patients $(n=386,65.3 \%)$ came from the central region of Uganda, where MNRH is located. At the time of presentation, $352(59.6 \%)$ presented due to RTA and 180 (30.5\%) due to assault. The median admission GCS score was 14 and the median LOS was 5 days. On examination, 25 (4.2\%) had abnormal pupils, 83 (14\%) had focal neural deficits, and 149 (25.2\%) had a CSF leak. Age at initial admission (mean 32.9 vs 32.5 years, $p=0.588$ ), sex $(88.3 \%$ vs $88.6 \%$ male, $\mathrm{p}=0.076$ ), and LOS (mean 7.6 vs 7.6 days, $\mathrm{p}=0.982)$ did not significantly differ between reachable 
TABLE 3. Longitudinal cohort characteristics

\begin{tabular}{|c|c|c|c|c|}
\hline Parameter & Respondents & Alive at Follow-Up & Deceased at Follow-Up & $p$ Value \\
\hline No. of patients & 591 & 538 & 53 & - \\
\hline \multicolumn{5}{|l|}{ Patient demographics } \\
\hline Sex & & & & 0.207 \\
\hline Female & $69(11.7)$ & $60(11.2)$ & $9(17)$ & \\
\hline Male & $522(88.3)$ & $478(88.9)$ & $44(83)$ & \\
\hline Median age (range), yrs & $28(18-95)$ & $28(18-90)$ & $35(18-95)$ & $<0.0001$ \\
\hline $18-30$ & $350(59.2)$ & $328(61)$ & $22(41.5)$ & \\
\hline $31-45$ & $149(25.2)$ & $136(25.3)$ & $13(24.5)$ & \\
\hline $46-60$ & $54(9.1)$ & $42(7.8)$ & $12(22.6)$ & \\
\hline $61+$ & $38(6.4)$ & $32(6)$ & $6(11.3)$ & \\
\hline Highest education level completed & & & & 0.901 \\
\hline None & $138(23.4)$ & $132(24.5)$ & $6(11.3)$ & \\
\hline Primary & $196(33.2)$ & $191(35.5)$ & $5(9.4)$ & \\
\hline Secondary & $4(0.68)$ & $4(0.7)$ & 0 & \\
\hline Diploma & $32(5.4)$ & $31(5.8)$ & $1(1.9)$ & \\
\hline Bachelor's degree & $22(3.7)$ & $21(3.9)$ & $1(1.9)$ & \\
\hline Unspecified & $199(33.7)$ & $159(29.6)$ & $40(75.5)$ & \\
\hline Employment status & & & & 0.248 \\
\hline Employed & $458(77.5)$ & $449(83.5)$ & $9(17)$ & \\
\hline Student & $28(4.7)$ & $28(5.2)$ & 0 & \\
\hline Unemployed & $64(10.8)$ & $60(11.2)$ & $4(7.6)$ & \\
\hline Unspecified & $41(6.9)$ & $1(0.2)$ & $40(75.5)$ & \\
\hline Region & & & & $<0.0001$ \\
\hline Central & $386(65.3)$ & $357(66.4)$ & $29(54.7)$ & \\
\hline Northern & $28(4.7)$ & $26(4.8)$ & $2(3.8)$ & \\
\hline Western & $108(18.3)$ & $100(18.6)$ & $8(15.1)$ & \\
\hline Eastern & $59(10)$ & $54(10)$ & $5(9.4)$ & \\
\hline Unspecified & $10(1.7)$ & $1(0.2)$ & $9(17.0)$ & \\
\hline \multicolumn{5}{|l|}{ Injury characteristics } \\
\hline Type of injury & & & & 0.454 \\
\hline RTA & $352(59.6)$ & $316(58.7)$ & $36(67.9)$ & \\
\hline Assault & $180(30.5)$ & $169(31.4)$ & $11(20.8)$ & \\
\hline Fall & $33(5.6)$ & $30(5.6)$ & $3(5.7)$ & \\
\hline Other & $8(1.4)$ & $7(1.3)$ & $1(1.9)$ & \\
\hline Unspecified & $18(3.1)$ & $16(3)$ & $2(3.8)$ & \\
\hline Median admission GCS score (range) & $14(4-15)$ & $14(4-15)$ & $12(4-15)$ & $<0.0001$ \\
\hline Positive CSF leak & $149(25.2)$ & $138(25.7)$ & $11(20.8)$ & 0.42 \\
\hline Abnormal pupil exam & $25(4.2)$ & $18(3.4)$ & $7(13.2)$ & 0.018 \\
\hline Focal neural deficits & $83(14)$ & $76(14.1)$ & $7(13.2)$ & 0.85 \\
\hline \multicolumn{5}{|l|}{ Inpatient management } \\
\hline Median LOS (range), days & $5(0-110)$ & $5(0-70)$ & $6(1-110)$ & 0.0002 \\
\hline \multicolumn{5}{|l|}{ Treatment } \\
\hline Ventilation & $5(0.85)$ & $4(0.7)$ & $1(1.9)$ & 0.45 \\
\hline Tracheostomy & $13(2.2)$ & $6(1.1)$ & $7(13.2)$ & $<0.0001$ \\
\hline Surgery & $130(22)$ & $114(21.2)$ & $16(30.2)$ & 0.15 \\
\hline Median discharge GCS score (range) & $15(8-15)$ & $15(10-15)$ & $15(8-15)$ & $<0.0001$ \\
\hline
\end{tabular}

Values are given as number of patients (\%) unless otherwise indicated. Boldface type indicates statistical significance. 
TABLE 4. Predictors of postdischarge mortality

\begin{tabular}{|c|c|c|c|c|}
\hline \multirow[b]{2}{*}{ Parameter } & \multicolumn{2}{|c|}{ Univariable } & \multicolumn{2}{|c|}{ Multivariable } \\
\hline & $\mathrm{HR}(95 \% \mathrm{Cl})$ & p Value & $\mathrm{HR}(95 \% \mathrm{Cl})$ & p Value \\
\hline \multicolumn{5}{|l|}{ Sex } \\
\hline \multicolumn{5}{|l|}{ Female (ref) } \\
\hline Male & $1.26(0.48-2.78)$ & 0.6 & & \\
\hline Age (continuous) & $1.04(1.02-1.05)$ & $<0.0001$ & $1.03(1.009-1.05)$ & 0.006 \\
\hline \multicolumn{5}{|l|}{ Region } \\
\hline \multicolumn{5}{|l|}{ Central (ref) } \\
\hline Other & $1.04(0.53-2.14)$ & 0.91 & & \\
\hline \multicolumn{5}{|l|}{ Injury } \\
\hline \multicolumn{5}{|l|}{ RTA (ref) } \\
\hline Non-RTA & $1.47(0.78-2.93)$ & 0.24 & & \\
\hline Admission GCS score (continuous) & $0.83(0.75-0.92)$ & 0.004 & $0.95(0.82-1.1)$ & 0.51 \\
\hline Positive CSF leak (vs no CSF leak) & $0.88(0.4-1.7)$ & 0.71 & & \\
\hline Abnormal pupil exam (vs normal pupil exam) & $0.28(0.12-0.82)$ & 0.02 & $0.99(0.3-3.78)$ & 0.99 \\
\hline Focal neural deficits (vs none) & $1.01(0.38-2.22)$ & 0.98 & & \\
\hline \multicolumn{5}{|l|}{ Inpatient management } \\
\hline LOS (continuous) & $1.03(1.01-1.04)$ & 0.002 & $0.99(0.97-1.02)$ & 0.91 \\
\hline Ventilation & $3.19(0.18-14.7)$ & 0.33 & & \\
\hline Tracheostomy & $10.4(3.92-22.9)$ & $<0.0001$ & $4.38(1.05-16.7)$ & 0.03 \\
\hline Surgery & $1.71(0.88-3.18)$ & 0.11 & & \\
\hline Discharge GCS score (continuous) & $0.61(0.51-0.73)$ & $<0.0001$ & $0.71(0.53-0.94)$ & 0.017 \\
\hline
\end{tabular}

and unreachable patients. Both admission and discharge GCS scores were similar between patients responding to follow-up calls $(n=591)$ and those who did not or were unreachable $(\mathrm{n}=381$; median admission GCS score 14 vs $14, \mathrm{p}=0.306$; median discharge GCS score 15 vs $15, \mathrm{p}=$ $0.805)$. Patients receiving surgery during the initial neurosurgical ward admission constituted $22 \%$ of patients (n $=130$ ) with longitudinal follow-up. Tracheostomies were required for 13 patients $(2.2 \%)$, while 5 patients $(0.8 \%)$ required mechanical ventilation during the initial inpatient stay.

Of the 591 patients with available postdischarge data, $53(9 \%)$ were deceased at follow-up (Table 3). Sex ( $\mathrm{p}=$ $0.207)$, type of injury at presentation $(p=0.454)$, presence of CSF leaks $(p=0.42)$, presence of focal neural deficits $(\mathrm{p}=0.85)$, mechanical ventilation $(\mathrm{p}=0.45)$, and receiving surgery $(\mathrm{p}=0.15)$ did not significantly differ between those alive and those deceased at follow-up. Patients who were alive at follow-up tended to have higher GCS scores at initial admission ( $\mathrm{p}<0.001)$, were less likely to have an abnormal diagnostic pupil examination $(\mathrm{p}=0.018)$, were less likely to have received a tracheostomy $(\mathrm{p}<0.001)$, and tended to have higher GCS scores at discharge $(\mathrm{p}<0.001)$. Older patients were also less likely to be alive at follow-up $(\mathrm{p}<0.001)$. Longer initial inpatient stays were observed in patients who were deceased at follow-up $(\mathrm{p}<0.001)$.

The median survival time was 17.8 months for all follow-up patients. Of the patients who were alive, the median follow-up time was 18.6 months, while the median survival for patients who were deceased at follow-up was
5 months (see Table 5). In univariable analyses, the admission GCS score (HR 0.83, 95\% CI 0.75-0.92), discharge GCS score (HR 0.61, 95\% CI 0.51-0.73), and abnormal pupil examination (HR $0.28,95 \%$ CI $0.12-0.82$ ) were associated with survival, while LOS (HR 1.03, 95\% CI 1.011.04), age (HR 1.04, 95\% CI 1.02-1.05), and tracheostomy (HR 10.4, 95\% CI 3.92-22.9) were associated with mortality (Table 4). Of these variables, age (HR 1.03, 95\% CI $1.009-1.05$ ), tracheostomy (HR 4.38, 95\% CI 1.05-16.7), and discharge GCS score (HR 0.71, 95\% CI 0.53-0.94) remained significant in multivariable analysis.

\section{Longitudinal Quality of Life}

Of the patients alive at follow-up, 443 (82.3\%) demonstrated good recovery (GOSE score 7-8), 71 (13.2\%) had moderate disability (GOSE score 5-6), and $22(4.1 \%)$ had severe disabilities (GOSE score 3-4; Table 5). The median GOSE score at the time of follow-up was 8 (range 3-8; the GOSE score was not available for 2 patients). Undergoing surgery was not associated with differences in long-term functional outcomes (surgical GOSE score 3-4, 7.1\%; 5-6, $12.4 \%$; 7-8, 80.5\%; nonsurgical GOSE score 3-4, 3.3\%; $5-6,13.5 \% ; 7-8,83.2 \% ; \mathrm{p}=0.212$, excluding 2 patients without GOSE scores). The majority of patients alive at follow-up responded that they were able to return to pre-TBI life, henceforth defined as a "return to normality" (455/538 patients, $84.6 \%$; Table 6). Of those not reaching full return to normality ( $\mathrm{n}=82 ; 1$ patient was unreachable), only 51 $(62.2 \%)$ cited a "near-total recovery of normal life," while the remaining patients denied a return to normality or near 
TABLE 5. Mortality-stratified cohort at long-term follow-up

\begin{tabular}{lccc}
\hline \multicolumn{1}{c}{ Parameter } & Respondents & Alive at Follow-Up & Deceased at Follow-Up \\
\hline No. of patients & 591 & 538 & 53 \\
\hline Follow-up outcome (range), mos & & & \\
\hline Median follow-up & $18.6(10.4-30.5)$ & $18.6(10.6-30.5)$ & $19.5(10.4-27.9)$ \\
\hline Median overall survival & $17.8(0.03-30.5)$ & $18.6(10.6-30.5)$ & $5(0.03-23.3)$ \\
\hline Median GOSE score (range) & $8(1-8)$ & $8(3-8)$ & $1(1-1)$ \\
\hline 3 & $13(2.2)$ & $13(2.4)$ & 0 \\
\hline 4 & $9(1.5)$ & $9(1.7)$ & 0 \\
\hline 5 & $26(4.4)$ & $26(4.8)$ & 0 \\
\hline 6 & $45(7.6)$ & $45(8.4)$ & 0 \\
\hline 7 & $42(7.1)$ & $42(7.8)$ & 0 \\
\hline 8 & $401(67.9)$ & $401(74.5)$ & $53(100)$ \\
\hline Deceased, GOSE score $=1$ & $53(9.0)$ & 0 & 0 \\
\hline Unknown & $2(0.3)$ & $2(0.4)$ & \\
\hline Val & & & 0 \\
\hline
\end{tabular}

Values are given as number of patients (\%) unless otherwise indicated.

normality. GOSE scores at follow-up correlated with return to pre-TBI life, with an average GOSE score of 7.7 in patients citing a return to normal life, 6.4 in patients citing a near-total recovery, and 4.9 in patients denying a return to normality $(\mathrm{p}<0.001)$. Notably, the percentage of patients experiencing depression and seizures was significantly lower in the group citing a return to normality than in the other two groups. Compared with crude rates of depression $(6.8 \%)$ and seizures $(4.0 \%)$ in patients reaching normality, $17.6 \%$ and $11.8 \%$ of patients attesting to a near return to normality experienced depression and seizures, respectively. In patients not achieving either normality or near normality, the rates of depression and seizures were $45.2 \%$ and $32.3 \%$, respectively.

Depression was frequent at follow-up, with 54 patients $(10.0 \%)$ noting depressive symptoms $(96.3 \%$ new-onset depression). Those experiencing depressive symptoms were equally likely to seek follow-up healthcare compared with those without depression (41/54 [75.9\%] vs 244/350 [69.7\%], $\mathrm{p}=0.351$ ). Patients experiencing at least one postdischarge seizure were more likely to pursue follow-up care than those not experiencing a seizure (29/33 [87.9\%] vs 308/505 [61.0\%], $\mathrm{p}=0.002$ ). Development of posttraumatic depression was not associated with differences in GCS score at admission (median score 13.5 vs $14, \mathrm{p}=$ 0.447 ). Patients presenting with severe TBI were at similar risk of developing seizures following discharge compared to those with nonsevere (mild and moderate) TBI (3/38 vs $31 / 500, p=0.725)$. Depression rates were also similar for severe and nonsevere TBI (6/38 vs 48/500, $\mathrm{p}=0.256)$.

The road distance and straight-line distance to MNRH based on district were available for 206 patients (34.9\%) with long-term follow-up (Table 7). On average, patients traveled a road distance of $73.4 \mathrm{~km}$ (average straight-line distance of $60.5 \mathrm{~km}$ ) prior to arrival at MNRH. The distance traveled varied greatly, with $39.3 \%(\mathrm{n}=81)$ of patients traveling more than $50 \mathrm{~km}$ and $29.1 \%(\mathrm{n}=60)$ of patients traveling more than $100 \mathrm{~km}$. Receiving surgery, inpatient mortality, and fraction of patients achieving full
TABLE 6. Long-term quality-of-life evaluation

\begin{tabular}{|c|c|c|c|c|}
\hline Parameter & Total & $\begin{array}{l}\text { Pursued } \\
\text { Follow-Up } \\
\text { Care }\end{array}$ & $\begin{array}{c}\text { No } \\
\text { Follow-Up } \\
\text { Care }\end{array}$ & $p$ Value \\
\hline No. of patients & $538(100)$ & $337(62.6)$ & $201(37.4)$ & \\
\hline Return to normal life & & & & 0.085 \\
\hline Yes & $455(84.6)$ & $280(83.1)$ & $175(87.1)$ & \\
\hline Almost & $51(9.5)$ & $32(9.5)$ & $19(9.5)$ & \\
\hline No & $31(5.8)$ & $25(7.4)$ & $6(3)$ & \\
\hline No answer & $1(0.2)$ & $0(0)$ & $1(0.5)$ & \\
\hline Depression & & & & 0.485 \\
\hline Yes, new onset & $52(9.7)$ & $39(11.6)$ & $13(6.5)$ & \\
\hline Yes, unchanged & $2(0.4)$ & $2(0.6)$ & $0(0)$ & \\
\hline No & $350(65.1)$ & $244(72.4)$ & $106(52.7)$ & \\
\hline No answer & $134(24.9)$ & $52(15.4)$ & $82(40.8)$ & \\
\hline Seizures & & & & 0.002 \\
\hline Yes & $33(6.1)$ & $29(8.6)$ & $4(2)$ & \\
\hline No & $504(93.7)$ & $308(91.4)$ & $196(97.5)$ & \\
\hline No answer & $1(0.2)$ & $0(0)$ & $1(0.5)$ & \\
\hline \multicolumn{5}{|l|}{ Has difficulty } \\
\hline Moving & $29(5.4)$ & $19(5.6)$ & $10(5)$ & 0.742 \\
\hline Seeing & $24(4.5)$ & $18(5.3)$ & $6(3)$ & 0.200 \\
\hline Hearing & $26(4.8)$ & $19(5.6)$ & $7(3.5)$ & 0.259 \\
\hline \multicolumn{5}{|l|}{ Needs assistance } \\
\hline Bathing & $6(1.1)$ & $4(1.2)$ & $2(1)$ & 0.838 \\
\hline Eating & $1(0.2)$ & $0(0)$ & $1(0.5)$ & 0.440 \\
\hline Using the toilet & $5(0.9)$ & $4(1.2)$ & $1(0.5)$ & 0.420 \\
\hline Dressing & $5(0.9)$ & $3(0.9)$ & $2(1)$ & 0.902 \\
\hline With hygiene & $12(2.2)$ & $8(2.4)$ & $4(2)$ & 0.771 \\
\hline Shopping & $23(4.3)$ & $17(5)$ & $6(3)$ & 0.253 \\
\hline Moving & $13(2.4)$ & $10(3)$ & $3(1.5)$ & 0.281 \\
\hline
\end{tabular}

Values are given as number of patients (\%). Boldface type indicates statistical significance. 
TABLE 7. Impact of preadmission transport distance $(n=206)$

\begin{tabular}{cc}
\hline & Value \\
\hline Distance summary & \\
\hline Road distance, $\mathrm{km}$ & $73.4(104.0)$ \\
\hline Mean (SD) & $14.47(1.84-496.93)$ \\
\hline Median (range) & $81(39.3)$ \\
\hline$>50 \mathrm{~km}, \mathrm{n}(\%)$ & $60(29.1)$ \\
\hline$>100 \mathrm{~km}, \mathrm{n}(\%)$ & $60.5(82.5)$ \\
\hline Straight-line distance, km & $12.71(1.26-352.19)$ \\
\hline Mean (SD) & $79(38.3)$ \\
\hline Median (range) & $59(28.6)$ \\
\hline$>50$ km, $\mathrm{n}(\%)$ & \\
\hline$>100$ km, $\mathrm{n}(\%)$ & $1.001(0.999-1.004), 0.208$ \\
\hline Distance metric* & $0.995(0.976-1.005), 0.476$ \\
\hline Road distance (continuous) & $0.999(0.997-1.001), 0.239$ \\
\hline Receiving surgery $\dagger$ & \\
\hline Inpatient mortality $\dagger$ & $1.002(0.999-1.005), 0.203$ \\
\hline Full recovery (GOSE) $\dagger$ & $0.995(0.973-1.006), 0.498$ \\
\hline Straight-line distance (continuous) & $0.998(0.996-1.001), 0.265$ \\
\hline Receiving surgery $\dagger$ &
\end{tabular}

${ }^{*}$ Data given as univariable OR $(95 \% \mathrm{Cl}), \mathrm{p}$ value.

† Dependent variable.

recovery were not associated with either road distance or straight-line distance (Table 7).

\section{Discussion}

Our study was designed to assess long-term clinical and functional outcomes in patients with TBI presenting to the MNRH neurosurgical ward, the main tertiary care center in Uganda. Utilizing phone-based surveillance, neurological and functional recovery were measured at a median of 1.5 years postdischarge. The primary aim of our study was to assess long-term neurobehavioral outcomes in Ugandan patients with TBI and investigate the relationships among initial patient presentation, in-hospital treatment course, and outpatient recovery from TBI.

\section{TBI in LMICs}

TBI incidence is a major cause of mortality and morbidity in LMICs. ${ }^{21,22}$ In particular, sub-Saharan Africa has seen steady increases in both TBI incidence and mortality. Postulated causes include population increases, prevalence of high-risk occupations, and rapid increases in motor vehicle transportation without stringent implementation of adequate traffic safety laws. ${ }^{15,23}$ Notably, RTAs and assaults constitute a significant portion of TBI cases in LMICs compared with HICs. ${ }^{9}$ Exacerbating the concerning increase in TBI burden in LMICs is the dearth of healthcare resources available for the populace. Until recently, 4 neurosurgeons managed the entirety of the Ugandan population, which recently exceeded 40 million. ${ }^{24}$ Operating rooms were understocked and understaffed; furthermore, outside of MNRH in the central region, Mbarara Regional Referral Hospital in the western region, and CURE Children's Hospital in the eastern region, neurosurgical services are difficult to access. In our cohort, patients traveled an average of $73.4 \mathrm{~km}$ (straight-line distance average of $60.5 \mathrm{~km}$ ), with nearly $30 \%$ of patients traveling more than $100 \mathrm{~km}$ to MNRH. Additionally, while TBI has been extensively studied in the context of HICs, understanding of TBI inhospital care and postdischarge outcomes remains limited in LMICs. As the burden of TBI continues to increase, understanding the factors associated with survival and longterm functional and neurological recovery is necessary to guide clinical decision-making and future targeted hospital development.

\section{Inpatient Mortality}

With limited inpatient resources available, inpatient mortality remains a significant concern in LMICs. In our study, the inpatient mortality rate was $23.7 \%$, with higher GCS score at admission, older patient age, female sex, and not receiving surgery contributing to increased risk of death. Although TBI cause (RTA vs assault) did not differ between those discharged and those who died as inpatients, it is likely that presurgical triage resulted in a differential rate of pursuing surgery, particularly if TBI severity differed based on TBI cause. In our cohort, TBI cause did emerge as being independently associated with receiving surgery, along with GCS score at the time of admission, and abnormal pupil examination. Of the patients who received surgery, 38 (15.7\%) died as inpatients; injury cause and GCS score at the time of admission remained associated with inpatient mortality. The finding that TBI associated with RTAs portends worse inpatient outcomes than assault-associated TBI, independent of GCS score, potentially reflects more extensive systemic injury or trauma. Regardless, given the high prevalence of TBI caused by RTAs in LMICs, this finding is concerning and reflects an urgent need to establish improved infrastructure for reducing RTA incidence.

Inpatient mortality after TBI can be caused by numerous factors, including malignant intracranial hypertension and intra- or postoperative complications such as infection and sepsis. ${ }^{25,26}$ As the hospital and operating room conditions in LMICs present unique challenges not frequently encountered in HICs, elucidating the factors associated with inpatient mortality is necessary to direct future quality improvement initiatives. It is notable that patients presenting with severe TBI, as defined by a GCS score of 3-8, were particularly susceptible to inpatient mortality at MNRH (52\%), compared with studies in HICs $(20 \%-39 \%) .{ }^{27-29}$ Additionally, despite its central location, extended transit times to MNRH likely preclude inclusion of many severe TBI cases who died either on-site or during transport. Given these considerations, the numbers reported in our study likely underrepresent the true burden of severe TBI in Uganda.

\section{Long-Term Survival and Functional Outcomes}

Long-term follow-up was available for $60.8 \%$ of patients, and we did not identify any admission characteris- 
tics systematically affecting follow-up likelihood. Factors identified in our study as being independently predictive of worse postdischarge mortality included older age, receipt of tracheostomy, lower discharge GCS score, and female sex. In addition to understanding predictors for postdischarge mortality, we sought to characterize long-term changes in functional and neurological recovery with an emphasis on retention of quality of life. Our study highlights that more than $15 \%$ of the admitted cohort demonstrated TBI-associated sequelae years after initial admission. Importantly, only $62.6 \%$ of patients had at least one healthcare visit between first discharge and our follow-up survey, and only $40.0 \%$ of patients accessed healthcare specifically for TBI follow-up. Given the dearth of healthcare access in the region and large distances and resources needed to travel, these values possibly reflect only cases in which postdischarge medical intervention was deemed necessary. As such, it is possible that less severe, yet burdensome sequelae are not captured. Because postdischarge monitoring of recovery is a critical element of the post-TBI recovery process in HICs, the lack of patient care in the years following TBI raises a number of questions.

Foremost among these questions is whether postdischarge mortality and morbidity could be reduced by regularly monitoring patient recovery after discharge from the hospital. Psychosocial, functional, and neurological recovery is an arduous process that, in HICs, involves regular physician visits and nonsurgical therapies. Seizures and depression are common after TBI and can result in lasting functional deficits. Posttraumatic epilepsy (PTE) is a known risk following TBI and is characterized by lateonset recurrent seizures (usually those occurring at least 24 hours following initial injury and frequently seen postdischarge) in the absence of additional CNS insult. ${ }^{30}$ Prior studies have established postdischarge seizure-associated mortality to be significantly elevated following TBI. ${ }^{31}$ In our study, $6.1 \%$ of patients demonstrated new-onset seizures after discharge, a number that likely underestimates the true incidence of PTE. Particularly in LMICs, where radiological monitoring and pharmaceutical intervention indicated for PTE can be lacking, PTE may incur significant postdischarge morbidity and mortality.

Similarly, posttraumatic depression is an often-underrecognized complication during the perioperative and postoperative recovery period that, especially in the absence of consistent psychiatric follow-up, presents unique problems in LMICs. In our cohort, we found that more than $10 \%$ of long-term TBI survivors self-recognized depressive symptoms following discharge. Prior incidence estimates of major depressive disorder after TBI vary, with some studies estimating 1-year postinjury prevalence to be approximately $13 \%$ and 5 -year postinjury prevalence to be approximately $50 \% .^{32,33}$ However, some studies have reported 6-month depression rates as high as $55 \%$, highlighting a need to better optimize assessment of post-TBI depression in LMIC settings. ${ }^{20}$ As the risk of posttraumatic depression continues to increase in the decade following injury, application of scalable methods to robustly assess patient recovery is necessary to understand the true burden of depression after TBI and to explore opportunities for intervention.

\section{Study Limitations}

Limitations of this study include factors associated with the longitudinal survey method, incomplete clinical follow-up, and logistical barriers including, but not limited to, language and culture. Unlike prior studies that have primarily relied on in-person follow-up visits to acquire longitudinal data, our study implements a more scalable phone-based method for assessing postdischarge recovery. However, it is possible that responses were biased based on patient condition or location. While discharge GCS scores between responding and nonresponding patients did not differ, it remains important to acknowledge that events following discharge could affect response rate. As is frequently encountered during research in LMICs, extensive inpatient clinical data were often unavailable. Notably, data on distance traveled to MNRH were only available for $34.9 \%$ of admitted patients, and additional studies are needed to robustly evaluate the effect of preadmission travel distance on outcomes. Granular details on clinical decision-making were unobtainable, making it difficult to ascertain reasons for which patients received certain procedures and services. Although post hoc analyses attempted to deconvolute the factors associated with receipt of surgery, it is possible that uncollected or unavailable data also played major roles in determining hospital management. Furthermore, although research coordinators conducting the phone interviews were well trained on the survey structure and interview format, comprehensive clinical data potentially obtainable during in-person physician visits were unavailable. Lastly, barriers to conducting robust data collection, such as language and cultural differences, must be considered. To attempt to address these concerns, the distinct cultural and social mannerisms associated with the projected cohort were considered during survey design; additionally, phone interviews were conducted in the patients' native languages by research coordinators trained in interview techniques. Nevertheless, miscommunication and errors during data collection remain a possibility. As many of the long-term outcomes assessed were subjective in nature, it is possible that screening bias remains; additional studies adjudicating our findings are necessary.

All patients included in this study were admitted to the neurosurgical ward, and our study does not include patients seen in the casualty/emergency department but not admitted to neurosurgery. As such, patients presenting with severe head injuries for whom neurosurgery was not considered may have been excluded from our study, presenting one potential source of bias. However, by considering only patients admitted to the neurosurgical ward, we also restrict our study cohort to a more homogenous TBI presentation, allowing for more clear interpretation of care protocols and outcomes. More broadly, while our study examines patterns of healthcare utilization, such as deployment of surgery, additional studies are needed to understand the causal factors driving healthcare patterns and practices in Uganda. Furthermore, long-term outcomes were assessed through a combination of GOSE scores and survey questions sourced from the Patient Health Questionnaire-9 (PHQ-9), 12-Item Short-Form Health Survey (SF-12), and Brief Illness Perception Questionnaire (IPQ9), based on their applicability to our study. However, these 
surveys have not been validated in the Ugandan setting and additional external validation is needed. Lastly, the mechanism of injury was not available; future analyses should consider subset analyses based on TBI etiology.

\section{Conclusions}

Neurological and functional sequelae following admission for TBI remain significant concerns in Uganda. Inpatient mortality is high and recovery from TBI is a lengthy process, with a significant number of patients not reaching self-perceived normality years after the injury. Neurological sequelae such as depression and seizures are common, especially in patients who did not return to their preinjury baseline after the TBI. Future work could establish a subset of the Ugandan neurosurgical population that might benefit most from prolonged functional monitoring.

\section{Acknowledgments}

We would like to acknowledge Doreen Nankunda and Evarest Ahaisibwe for their assistance in data collection. Funding for this study was provided by the Stanford Medical Scholars Fellowship Program and the Stanford Society of Physician Scholars fund.

\section{Appendix}

\section{Long-Term Variables Assessed}

All longitudinal follow-up surveys were constructed using elements from the PHQ-9, SF-12, and IPQ-9. All questions were translated into the local language with assistance from research staff and collaborators located at MNRH. Variables included in our analyses are as follows:

\section{General}

1. District of residence

2. Needs caregiver?

3. Prior employment status

4. Current employment status

5. Received postdischarge care

a. Reason for seeking postdischarge care

\section{Day-to-Day Activities}

6. Any difficulty moving?

7. Any difficulty seeing?

8. Any difficulty hearing?

9. Require assistance eating?

10. Require assistance using the toilet?

11. Require assistance dressing?

12. Require assistance with hygiene?

13. Require assistance shopping?

14. Require assistance moving locally?

\section{Seizures and Depression}

15. Any symptoms of depression?

a. Yes-new after TBI

b. Yes-unchanged

c. No

16. Any seizures since discharge?

a. Yes

b. No

\section{References}

1. Appenteng R, Nelp T, Abdelgadir J, et al. A systematic re- view and quality analysis of pediatric traumatic brain injury clinical practice guidelines. PLoS One. 2018;13(8):e0201550.

2. De Silva MJ, Roberts I, Perel P, et al. Patient outcome after traumatic brain injury in high-, middle- and low-income countries: analysis of data on 8927 patients in 46 countries. Int J Epidemiol. 2009;38(2):452-458.

3. Hyder AA, Wunderlich CA, Puvanachandra P, et al. The impact of traumatic brain injuries: a global perspective. NeuroRehabilitation. 2007;22(5):341-353.

4. Tran TM, Fuller AT, Kiryabwire J, et al. Distribution and characteristics of severe traumatic brain injury at Mulago National Referral Hospital in Uganda. World Neurosurg. 2015;83(3):269-277.

5. Hukkelhoven CW, Steyerberg EW, Farace E, et al. Regional differences in patient characteristics, case management, and outcomes in traumatic brain injury: experience from the tirilazad trials. J Neurosurg. 2002;97(3):549-557.

6. Carlozzi NE, Tulsky DS, Kisala PA. Traumatic brain injury patient-reported outcome measure: identification of healthrelated quality-of-life issues relevant to individuals with traumatic brain injury. Arch Phys Med Rehabil. 2011;92(10) (suppl):S52-S60.

7. Whiteneck GG, Gerhart KA, Cusick CP. Identifying environmental factors that influence the outcomes of people with traumatic brain injury. J Head Trauma Rehabil. 2004;19(3):191-204.

8. Jayaraman S, Ozgediz D, Miyamoto J, et al. Disparities in injury mortality between Uganda and the United States: comparative analysis of a neglected disease. World J Surg. 2011;35(3):505-511.

9. Dewan MC, Rattani A, Gupta S, et al. Estimating the global incidence of traumatic brain injury. J Neurosurg. 2019;130(4):1080-1097.

10. Kuo BJ, Vaca SD, Vissoci JRN, et al. A prospective neurosurgical registry evaluating the clinical care of traumatic brain injury patients presenting to Mulago National Referral Hospital in Uganda. PLoS One. 2017;12(10):e0182285.

11. Xu LW, Vaca SD, Nalwanga J, et al. Life after the neurosurgical ward in sub-Saharan Africa: neurosurgical treatment and outpatient outcomes in Uganda. World Neurosurg. 2018;113:e153-e160.

12. Vaca SD, Kuo BJ, Nickenig Vissoci JR, et al. Temporal delays along the neurosurgical care continuum for traumatic brain injury patients at a tertiary care hospital in Kampala, Uganda. Neurosurgery. 2019;84(1):95-103.

13. Fuller A, Tran T, Muhumuza M, Haglund MM. Building neurosurgical capacity in low and middle income countries. eNeurologicalSci. 2015;3:1-6.

14. Haglund MM, Fuller AT. Global neurosurgery: innovators, strategies, and the way forward. J Neurosurg. 2019;131(4):993-999.

15. Mehmood A, Zia N, Hoe C, et al. Traumatic brain injury in Uganda: exploring the use of a hospital based registry for measuring burden and outcomes. BMC Res Notes. 2018;11(1):299.

16. Zia N, Mehmood A, Namaganda RH, et al. Causes and outcomes of traumatic brain injuries in Uganda: analysis from a pilot hospital registry. Trauma Surg Acute Care Open. 2019;4(1):e000259.

17. Vaca SD, Xu LW, Nalwanga J, et al. Long-term follow-up of pediatric head trauma patients treated at Mulago National Referral Hospital in Uganda. J Neurosurg Pediatr. 2018;23(1):125-132.

18. Xu LW, Vaca SD, He JQ, et al. Neural tube defects in Uganda: follow-up outcomes from a national referral hospital. Neurosurg Focus. 2018;45(4):E9.

19. Jennett B, Bond M. Assessment of outcome after severe brain damage. Lancet. 1975;1(7905):480-484.

20. Bangirana P, Giordani B, Kobusingye O, et al. Patterns of 
traumatic brain injury and six-month neuropsychological outcomes in Uganda. BMC Neurol. 2019;19(1):18.

21. Ghajar J. Traumatic brain injury. Lancet. 2000;356(9233): 923-929.

22. Maas AIR, Menon DK, Adelson PD, et al. Traumatic brain injury: integrated approaches to improve prevention, clinical care, and research. Lancet Neurol. 2017;16(12):987-1048.

23. James SL, Theadom A, Ellenbogen RG, et al. Global, regional, and national burden of traumatic brain injury and spinal cord injury, 1990-2016: a systematic analysis for the Global Burden of Disease Study 2016. Lancet Neurol. 2019;18(1):56-87.

24. Haglund MM, Warf B, Fuller A, et al. Past, present, and future of neurosurgery in Uganda. Neurosurgery. 2017;80(4):656-661.

25. Corral L, Javierre CF, Ventura JL, et al. Impact of nonneurological complications in severe traumatic brain injury outcome. Crit Care. 2012;16(2):R44.

26. Osborn TM, Tracy JK, Dunne JR, et al. Epidemiology of sepsis in patients with traumatic injury. Crit Care Med. 2004;32(11):2234-2240.

27. Dawes AJ, Sacks GD, Cryer HG, et al. Intracranial pressure monitoring and inpatient mortality in severe traumatic brain injury: a propensity score-matched analysis. J Trauma Acute Care Surg. 2015;78(3):492-502.

28. Farahvar A, Gerber LM, Chiu YL, et al. Increased mortality in patients with severe traumatic brain injury treated without intracranial pressure monitoring. J Neurosurg. 2012;117(4):729-734.

29. Prabhakaran K, Petrone P, Lombardo G, et al. Mortality rates of severe traumatic brain injury patients: impact of direct versus nondirect transfers. J Surg Res. 2017;219:66-71.

30. Ding K, Gupta PK, Diaz-Arrastia R. Epilepsy after traumatic brain injury. In: Laskowitz D, Grant G, eds. Translational Research in Traumatic Brain Injury. CRC Press; 2016.
31. Ventura T, Harrison-Felix C, Carlson N, et al. Mortality after discharge from acute care hospitalization with traumatic brain injury: a population-based study. Arch Phys Med Rehabil. 2010;91(1):20-29.

32. Deb S, Lyons I, Koutzoukis C, et al. Rate of psychiatric illness 1 year after traumatic brain injury. Am J Psychiatry. 1999;156(3):374-378.

33. van Reekum R, Bolago I, Finlayson MA, et al. Psychiatric disorders after traumatic brain injury. Brain Inj. 1996;10(5):319-327.

\section{Disclosures}

Dr. Haglund reports clinical or research support for this study from Nuva and LifeNet, support of non-study-related clinical or research effort from UCB Pharma, and being a consultant to Nuva.

\section{Author Contributions}

Conception and design: Jin, Kakusa, Ku, Grant. Acquisition of data: all authors. Analysis and interpretation of data: Jin, Kakusa, Ku, Fuller, Grant. Drafting the article: Jin, Kakusa. Critically revising the article: $\mathrm{Ku}$, Vaca, Xu, Fuller, Haglund, Grant. Reviewed submitted version of manuscript: all authors. Approved the final version of the manuscript on behalf of all authors: Jin. Statistical analysis: Jin, Kakusa. Administrative/technical/material support: Haglund, Grant. Study supervision: Haglund, Grant.

\section{Correspondence}

Gerald A. Grant: Stanford University, Stanford, CA. ggrant2@ stanford.edu. 